\title{
The Patient Global Assessment of Disease Activity in Rheumatoid Arthritis: Identification of Underlying Latent Factors
}

\author{
Divya N. V. Challa - Cynthia S. Crowson · John M. Davis III
}

Received: March 23, 2017 / Published online: May 9, 2017

(C) The Author(s) 2017. This article is an open access publication

\begin{abstract}
Introduction: The patient global assessment of disease activity is a crucial component of various measures of disease activity in rheumatoid arthritis (RA). Our objective was to identify underlying latent traits driving the patient global assessment using a quantitative, multivariable data reduction approach.

Methods: This was a prospective cross-sectional study of consecutive patients with RA. The patient sample was stratified to include 50 patients with patient-provider discordance (i.e., at least $25-\mathrm{mm}$ absolute difference between the patient and provider global assessments) and 20 patients with patient-provider concordance (i.e., less than 25-mm absolute difference between the patient and provider global assessments). Data were collected from the most recent rheumatology visit, including patient characteristics, current RA medications, and comorbidities. Participants
\end{abstract}

Enhanced content To view enhanced content for this article go to www.medengine.com/Redeem/1A18F0604 FFE021C.

D. N. V. Challa · J. M. Davis III (ه)

Division of Rheumatology, Department of

Medicine, Mayo Clinic, Rochester, MN, USA

e-mail: davis.john4@mayo.edu

\section{S. Crowson}

Division of Biostatistics and Informatics, Department of Health Sciences Research, Mayo

Clinic, Rochester, MN, USA completed several validated patient-reported outcome measures. The data were evaluated using factor analysis, and then linear regression was used to determine the variability in the patient global assessment explained by the factor scores. Results: The study included 70 patients with mean age of 61 years, $73 \%$ female, and with mean disease duration of 8 years. The means (SD) for the patient and provider global assessments were 44.6 (22.7) and 20.1 (17.7), respectively. Factor analysis yielded eight factors that represented measurements of pain, fatigue, depression or anxiety symptoms, prior diagnosis of depression or anxiety, advanced age and degenerative arthritis, inability to participate, fibromyalgia (clinical diagnosis and Widespread Pain Index), and undetermined. Linear regression analysis showed that fibromyalgia explained the greatest proportion of the variance in the patient global assessment followed by the other factors.

Conclusion: Latent factors underlying the patient global assessment include pain, depression and anxiety, inability to participate, fibromyalgia, advanced age, and degenerative arthritis.

Keywords: Disease activity; Discordance; Rheumatoid arthritis

\section{INTRODUCTION}

The patient global assessment (global assessment) of disease activity is a crucial component 
of validated disease activity scores, response criteria, and the American College of Rheumatology (ACR)/European League Against Rheumatism (EULAR) definition of remission in rheumatoid arthritis (RA). Patients rate their global assessment on a visual analog scale (VAS) by answering the question, "Considering all of the ways your disease affects you, how well are you doing in the past week?" Hence, the patient global not only assesses disease from the patient perspective but also encompasses various factors affecting patients in addition to RA.

Many studies have shown that pain is the strongest factor driving the patient global assessment [1-5]. Several studies have shown that depression affects the patient global assessment, although extensive study has not been done to determine the effect of other psychological factors [1, 3, 6-8]. Depression and other psychological factors might influence patients' assessments of their disease activity; hence, further work is necessary to determine the various biomedical and biopsychosocial factors underlying the patient global assessment.

Recent work has revealed that $30 \%$ of patients with RA suffer from persistent undifferentiated symptoms of pain, fatigue, and functional disability despite seeming attainment of low inflammatory disease activity [9]. This discordance in assessments of disease activity between patients and their providers is a critical issue, so addressing factors that influence patients' assessments of disease activity is necessary to narrow this gap and improve patient outcomes. The purpose of this study was to identify the underlying latent factors driving the patient global assessment using a quantitative, multivariable data reduction approach.

\section{METHODS}

\section{Study Design and Populations}

A prospective cross-sectional study was conducted at Mayo Clinic in Rochester, Minnesota. A total of 70 consecutive patients were recruited from our outpatient rheumatology practice with a clinical diagnosis of RA fulfilling the
ACR/EULAR 2010 classification criteria [10]. Eligibility required age to be at least 18 years, residence within 150 miles of the clinic, at least two outpatient follow-up appointments in the prior 18 months, and the most recent appointment within 4 weeks preceding study enrollment. Enrollment was stratified into two groups: one with patient-provider discordance (i.e., at least $25-\mathrm{mm}$ absolute difference between the patient and provider global assessments, which has been used as a clinically important difference by a previous study [11]), and the other with patient-provider concordance (less than $25-\mathrm{mm}$ absolute difference between the patient and provider global assessments). Recruitment of the group with patient-provider concordance was stratified to include 10 patients with low disease activity and 10 with moderate to high disease activity. The Mayo Clinic Institutional Review Board (IRB) approved the study. All patients provided written informed consent.

\section{Data Collection}

Data were collected from the electronic medical records for the most recent rheumatologic visit prior to the research study visit, including patient demographics, patient and provider global assessment of disease activity (0-100), tender and swollen joint counts (0-28), inflammatory markers (i.e., erythrocyte sedimentation rate, C-reactive protein), use of traditional and biologic disease-modifying antirheumatic drugs (DMARDs), use of anxiolytics or antidepressants, opioids, fibromyalgia pain medications, and sleep aids. All prior medical records were used to abstract data including the duration of rheumatoid arthritis, clinical diagnosis of comorbidities like anxiety, depression, fibromyalgia, osteoarthritis, and radiographic evidence of joint erosions in hands and feet, serologies for rheumatoid factor (RF) and anti-cyclic citrullinated antibodies (anti-CCP).

\section{Patient-Reported Outcomes (PROs)}

All participants completed several questionnaires at a research study visit, which included 
physical and psychological domains. Physical domains included the pain VAS, Short-Form McGill Pain Questionnaire-2 (SF-MPQ-2), Health Assessment Questionnaire-II (HAQ-II), Fibromyalgia Research Survey, and Bristol Rheumatoid Arthritis Fatigue (BRAF) score. SF-MPQ-2 consists of 15 pain descriptors, including six continuous (e.g., aching or throbbing), six intermittent (e.g., stabbing or piercing), six neuropathic (e.g., hot-burning or numbness), and four affective (e.g., punishing or cruel, fearful), which are rated on an intensity scale of $0-3[12,13]$. Five pain scores are derived from the sum of the intensity rank values of the words chosen for continuous, intermittent, neuropathic, affective, and total descriptors. HAQ-II is a widely used, reliable, and valid 10 -item questionnaire that measures functional status [14]. The Fibromyalgia Research Survey includes the Widespread Pain Index (WPI) and Symptom Severity (SS). WPI is a measure of the number of painful body regions, and the SS score enables assessment of key somatic domains, including fatigue, unrefreshing sleep, concentration, and other somatic symptoms in persons with current or previous fibromyalgia [15]. The polysymptomatic distress scale (PSD), the sum of the WPI and SS, characterizes "fibromyalgia-ness" on a continuous scale [16]. The BRAF score assesses numeric rating scales for fatigue severity, effect, and coping during the past 7 days. All three dimensions are rated from 0 to 10 , and the total score is obtained by dividing the sum of individual scores by 3 [17].

Psychological domains included the Patient Health Questionnaire (PHQ-9), Generalized Anxiety Disorder (GAD-7), Mindfulness Attention and Awareness Scale (MAAS), and several Patient-Reported Outcome Measure Information System (PROMIS) forms. PHQ-9 is a reliable and valid measure of depression severity that scores each of the nine DSM-IV criteria as 0 (not at all) to 3 (nearly every day) [18]. The IRB-approved protocol required that patients with PHQ-9 scores of at least 10 be evaluated by the investigator and given the option of psychiatry referral. GAD-7 is a seven-item scale used to measure generalized anxiety symptoms. Each item is rated from 0 to 3 ("not at all" to "nearly every day") and all the item scores are added to get the total score [19]. MAAS is a 15-item scale designed to assess core characteristics of dispositional mindfulness and attention. The scale shows strong psychometric properties and taps a unique quality of consciousness that is related to and predictive of a variety of self-regulation and well-being constructs [20]. The PROMIS short forms for pain interference (version 1.0); sleep disturbance (version 1.0); fatigue (version 1.0 ), and ability to participate in social roles and activities (version 2.0) were assessed. Each of the PROMIS instruments has eight items, rated by the patients as $1-5$, and total scores are obtained for each of the four instruments separately.

\section{Statistical Methods}

Data were evaluated using exploratory factor analysis as a data reduction method to determine the latent constructs in the data. Linear regression models were then used to determine the variability in the patient global assessment explained by the factor scores. A heat map was used to depict the factor loadings. Analyses were performed using SAS version 9.4 (SAS Institute, Cary, NC, USA) and R 3.2.0 (R Foundation for Statistical Computing, Vienna, Austria).

\section{RESULTS}

The study included a total of 70 patients with mean (SD) age of 61.0 (13.6) years, and 73\% were female (Table 1 ). Nearly all of the patients were non-Hispanic white (99\%). The mean (SD) disease duration was 8.0 (6.7) years. Overall, $53 \%$ were $\mathrm{RF}$ and/or anti-CCP positive. The mean (SD) for the patient global assessment was 44.3 (22.6) and for the provider global assessment was 20.1 (17.7). Among the group of 50 patients with patient-provider discordance, 46 patients had positive discordance (i.e., patient global assessment at least $25 \mathrm{~mm}$ higher the provider global assessment); the remaining four patients had negative discordance (i.e., patient global assessment more than $25 \mathrm{~mm}$ lower than the patient global assessment). The remaining 20 patients had patient-provider concordance. 
Table 1 Characteristics of 20 concordant and 50 discordant study subjects

\begin{tabular}{|c|c|c|c|}
\hline Characteristic & $\begin{array}{l}\text { Concordant } \\
(n=20)\end{array}$ & $\begin{array}{l}\text { Discordant } \\
(n=50)\end{array}$ & $p$ value \\
\hline Age, years, mean (SD) & $56.4(13.7)$ & $62.8(13.3)$ & 0.09 \\
\hline Sex, female & $14(70 \%)$ & $37(74 \%)$ & 0.73 \\
\hline Race, non-Hispanic white & $18(90 \%)$ & $49(98 \%)$ & 0.14 \\
\hline Disease duration, years, mean (SD) & $7.8(5.1)$ & $8.1(7.3)$ & 0.70 \\
\hline Time from clinical visit to study visit, days, mean (SD) & $21.5(14.4)$ & $17.6(6.1)$ & 0.11 \\
\hline \multicolumn{4}{|l|}{ Disease assessments } \\
\hline Patient global $(0-100 \mathrm{~mm})$ & $16(6,48)$ & $52(43,62)$ & $<0.001$ \\
\hline Provider global $(0-100 \mathrm{~mm})$ & $22(5,50)$ & $12(10,25$ & 0.36 \\
\hline Pain $(0-100 \mathrm{~mm})$ & $22(10,62)$ & $57(42,71)$ & 0.003 \\
\hline DAS28-CRP & $2.0(1.8,3.4)$ & $3.1(2.5,3.5)$ & 0.14 \\
\hline HAQ disability index, mean (SD) & $0.5(0.5)$ & $0.9(0.6)$ & 0.009 \\
\hline \multicolumn{4}{|l|}{ Laboratory assessments } \\
\hline $\mathrm{CRP}, \mathrm{mg} / \mathrm{L}$ & $<3.0(<3.0,4.5)$ & $<3.0(<3.0,5.8)$ & 0.72 \\
\hline RF, positive & $14(70 \%)$ & $22(45 \%)$ & 0.07 \\
\hline ACPA, positive & $11(65 \%)$ & $22(49 \%)$ & 0.26 \\
\hline \multicolumn{4}{|l|}{ Medication use } \\
\hline Prednisone & $6(30 \%)$ & $19(38 \%)$ & 0.53 \\
\hline cDMARD & $19(95 \%)$ & $43(86 \%)$ & 0.28 \\
\hline bDMARD & $4(20 \%)$ & $17(34 \%)$ & 0.25 \\
\hline Change of RA medication or dosage at most recent clinical visit & $5(25 \%)$ & $6(12 \%)$ & 0.18 \\
\hline
\end{tabular}

Values are number (\%), unless otherwise specified

$R F$ rheumatoid factor, $A C P A$ anti-cyclic citrullinated peptide antibody, DAS28-CRP disease activity score in 28 joints using C-reactive protein, $C R P$ C-reactive protein, $c D M A R D$ conventional disease-modifying antirheumatic drug, $b D M A R D$ biologic disease-modifying antirheumatic drug

The analysis yielded eight latent factors underlying the patient global assessment (Fig. 1). Factor 1 included the SF-MPQ (total, sensory and affective) scores, SF-MPQ current pain intensity, pain VAS, and PROMIS pain interference, clearly representing pain as a complex domain. Factor 2 comprised PROMIS fatigue and the BRAF, clearly representing fatigue. Anxiousness by the GAD-7 and depressed mood symptoms by the PHQ-9 scores were strong components of factor 3 , while clinical diagnoses of anxiety and depression dominated in factor 4, notably separate from the patient-reported measures of these domains. Aging and presence of osteoarthritis defined factor 5. Clinical diagnosis of fibromyalgia and fibromyalgia research survey WPI were strong components in factor 7.

PROMIS ability to participate in social roles and activities was a strongly negative component in factor 6, which makes sense as high scores indicate better health and low scores indicate poor health. This score was also a strongly negative component of factor 1 , which 


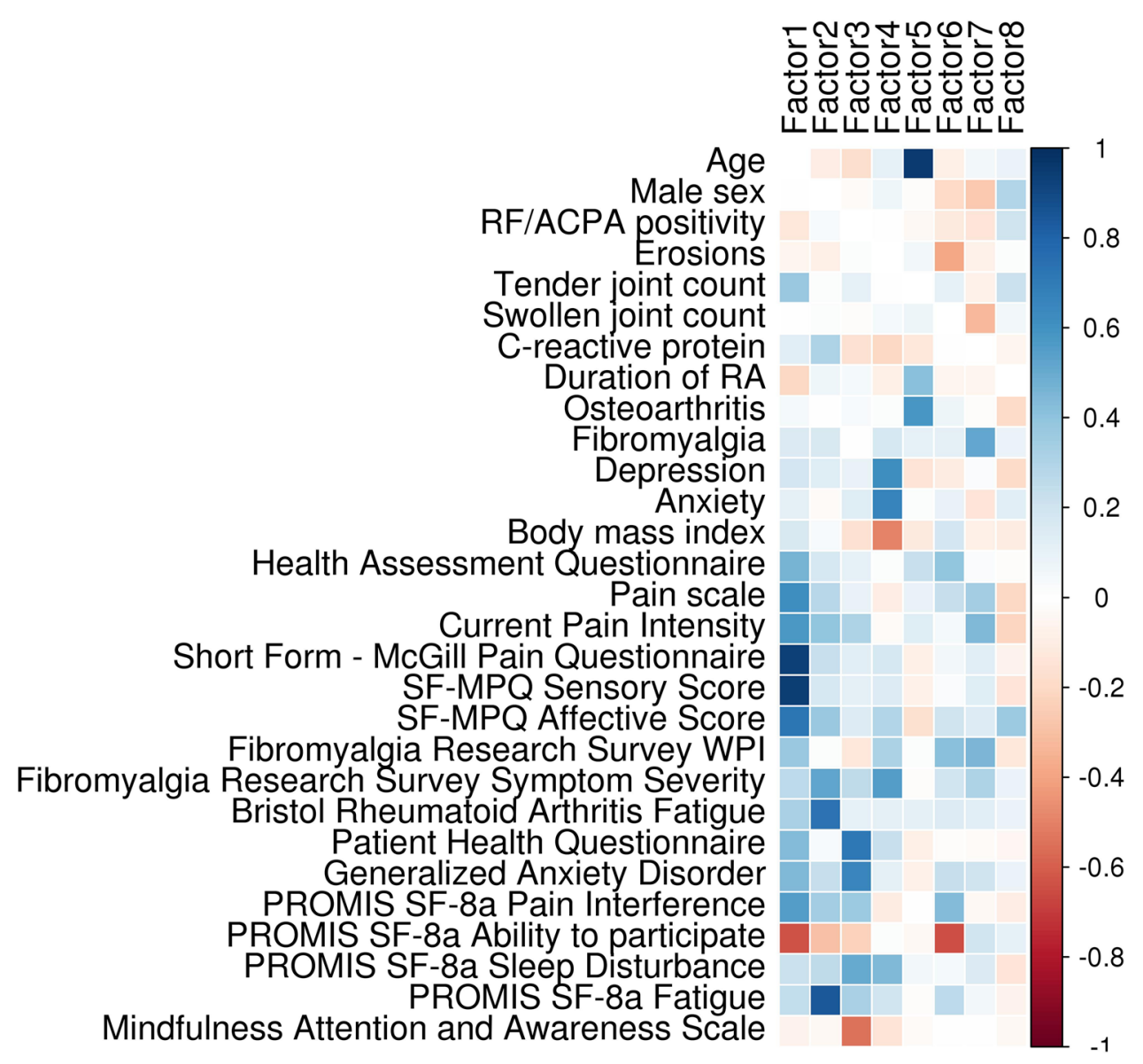

Fig. 1 Factor analysis of rheumatoid arthritis disease characteristics, associated comorbidities, and patient-reported outcomes in rheumatoid arthritis. Dark colored blocks in each column indicate the strongest characteristics in each principal component. Blue color indicates positive loading and orange indicates negative loading. Factor 1

included other measures of pain. MAAS was a strong negative component of factor 3, which again makes sense as high MAAS scores indicate more mindfulness and factor 3 appears to measure psychological distress. However, body mass index was a strong negative component of factor 4 , which is unclear because factor 4 contains clinical diagnoses of depression and anxiety. There were no strong factors (i.e., loadings greater than 0.4 ) identified in factor 8 . The strongest loading of factor 8 was the affective score of the SF-MPQ (loading $=0.36$ ). All eight factors together explained $58 \%$ of the variability in the RA disease characteristics, associated comorbidities, and PROs evaluated. pain; Factor 2 fatigue; Factor 3 psychological distress; Factor 4 depression and anxiety by clinical diagnosis; Factor 5 advanced age and degenerative arthritis; Factor 6 inability to participate; Factor 7 fibromyalgia (Widespread Pain Index and clinical diagnosis); Factor 8 unknown

The first factor explained $17 \%$ and the last factor explained only $2 \%$ of the variance in the patient global assessment.

Linear regression analysis showed that the factors representing fibromyalgia (factor 7), inability to participate in social roles and activities (factor 6), and pain (factor 1) showed the greatest correlation with patient global assessment followed by psychological distress (factor 3) and advanced age and degenerative arthritis (factor 5) (Table 2). Of note, fatigue and clinical diagnoses of depression or anxiety were not significantly associated with patient global assessment. Univariable modeling showed that fibromyalgia explained the greatest variability 
Table 2 Univariable and multivariable associations between identified latent factors and the patient global assessment in 70 patients with rheumatoid arthritis

\begin{tabular}{|c|c|c|c|c|c|}
\hline \multirow[t]{2}{*}{ Factor } & \multicolumn{3}{|c|}{ Univariable models } & \multicolumn{2}{|c|}{ Multivariable model* } \\
\hline & $\overline{\text { Coefficient (SE) }}$ & $p$ value & $\overline{\text { Adjusted } R^{2}}$ & $\overline{\text { Coefficient (SE) }}$ & $p$ value \\
\hline Factor 1: pain & $6.34(2.88)$ & 0.032 & 0.063 & $6.67(2441)$ & 0.011 \\
\hline Factor 2: fatigue & $2.20(2.94)$ & 0.46 & 0.0 & $2.81(2.49)$ & 0.26 \\
\hline Factor 3: psychological distress & $4.10(2.69)$ & 0.13 & 0.022 & $4.49(2.32)$ & 0.059 \\
\hline $\begin{array}{l}\text { Factor 4: depression and anxiety (by clinical } \\
\text { diagnosis) }\end{array}$ & $0.44(2.70)$ & 0.87 & 0.0 & $0.75(2.28)$ & 0.74 \\
\hline Factor 5: advanced age and degenerative arthritis & $4.67(2.94)$ & 0.12 & 0.026 & $4.62(2.52)$ & 0.073 \\
\hline Factor 6: inability to participate & $6.50(2.76)$ & 0.022 & 0.074 & $6.19(2.44)$ & 0.014 \\
\hline Factor 7: fibromyalgia & $6.14(2.56)$ & 0.020 & 0.077 & $5.78(2.27)$ & 0.014 \\
\hline Factor 8: unknown & $-3.57(2.88)$ & 0.22 & 0.009 & $-3.54(2.46)$ & 0.16 \\
\hline
\end{tabular}

$S E$ standard error

* Adjusted $R^{2}=0.28$

in the patient global assessment (adjusted $\left.R^{2}=0.077\right)$. Multivariable analysis showed that the factors altogether explained $28 \%$ of the variability in the patient global assessment.

\section{DISCUSSION}

The patient global assessment is a crucial and widely used measure of disease activity for RA. This study is the first to identify latent factors driving the patient global assessment using factor analysis. Pain, psychological distress, advanced age and degenerative arthritis, inability to participate, and fibromyalgia are key drivers of patient global assessment in patients with RA. These factors altogether explained $28 \%$ of the variability in the patient global assessment.

Several studies showed that pain is the strongest correlate of the patient global assessment [1-5]. It was the second most important factor underlying the patient global assessment in our study following fibromyalgia. However, fibromyalgia and other coexisting conditions have a substantial influence on patients' perceptions of pain. These findings highlight pain as a major factor affecting the patients' perceptions of disease activity. Our study also found that other physical domains like degenerative arthritis and advanced age significantly affected patient global assessment.

Studies have shown that depression can influence patients' assessment of disease activity [1, 3, 6-8]. Our study also showed that psychological distress (symptoms of anxiety and depression) affected patient global assessment. Psychological distress might measure the emotional significance of the disease better than clinical diagnosis of depression or anxiety [21]. We also found that clinical diagnosis of anxiety and/or depression affected patient global assessment similar to some previous studies [1, 3, 6-8]. In addition, inability to participate in social roles and activities also affected the patient global assessment.

Limitations of our study include its limited sample size and the inherent limitations of the patient global assessment measured by VAS, as patients tend to perceive their disease activity on the basis of associated comorbidities and not just RA [21]. Patients answered numerous questionnaires, which were time-consuming, so we cannot rule out bias due to questionnaire burden.

\section{CONCLUSIONS}

The results of this study suggest that rheumatologists should take into consideration all of 
the identified drivers of patient global assessment when managing patients with RA. Systematic assessment of these domains using validated patient-reported outcome measures could improve shared decision-making and outcomes of RA. Future studies are necessary to determine how to efficiently and effectively measure these factors in routine practice settings. Evidenced-based approaches are needed targeting specific domains to improve patients' quality of life and functional status. These efforts are anticipated to improve communication between patients and providers and optimize disease outcomes.

\section{ACKNOWLEDGEMENTS}

The Mayo Foundation Eaton Family Career Development Award in Innovative Rheumatoid Arthritis Research and the CTSA Grant Number UL1 TR000135 from the National Center for Advancing Translational Science (NCATS) supported this study. Its contents are solely the responsibility of the authors and do not necessarily represent the official views of the NIH. No sponsorship was provided for the study and article processing charges.

All named authors meet the International Committee of Medical Journal Editors (ICMJE) criteria for authorship for this manuscript, take responsibility for the integrity of the work as a whole, and have given final approval for the version to be published.

Disclosures. Divya N. V. Challa, Cynthia S. Crowson, and John M. Davis III have nothing to disclose.

Compliance with Ethics Guidelines. The Mayo Clinic Institutional Review Board approved the study. All patients provided written informed consent.

Data Availability. The datasets analyzed during the current study are available from the corresponding author on reasonable request.
Open Access. This article is distributed under the terms of the Creative Commons Attribution-NonCommercial 4.0 International License (http://creativecommons.org/licenses/ by-nc/4.0/), which permits any noncommercial use, distribution, and reproduction in any medium, provided you give appropriate credit to the original author(s) and the source, provide a link to the Creative Commons license, and indicate if changes were made.

\section{REFERENCES}

1. Khan NA, Spencer HJ, Abda EA, et al. Patient's global assessment of disease activity and patient's assessment of general health for rheumatoid arthritis activity assessment: are they equivalent? Ann Rheum Dis. 2012;71(12):1942-9.

2. Markenson JA, Koenig AS, Feng JY, et al. Comparison of physician and patient global assessments over time in patients with rheumatoid arthritis: a retrospective analysis from the RADIUS cohort. J Clin Rheumatol. 2013;19(6):317-23.

3. Smedstad LM, Kvien TK, Moum T, Vaglum P. Correlates of patients' global assessment of arthritis impact. A 2-year study of 216 patients with RA. Scand J Rheumatol. 1997;26(4):259-65.

4. Studenic P, Radner H, Smolen JS, Aletaha D. Discrepancies between patients and physicians in their perceptions of rheumatoid arthritis disease activity. Arthritis Rheum. 2012;64(9):2814-23.

5. Ward MM, Leigh JP. The relative importance of pain and functional disability to patients with rheumatoid arthritis. J Rheumatol. 1993;20(9):1494-9.

6. Cordingley L, Prajapati R, Plant D, et al. Impact of psychological factors on subjective disease activity assessments in patients with severe rheumatoid arthritis. Arthritis Care Res (Hoboken). 2014;66(6):861-8.

7. Inanc $\mathrm{N}$, Yilmaz-Oner $\mathrm{S}$, Can $\mathrm{M}$, Sokka $\mathrm{T}$, Direskeneli $\mathrm{H}$. The role of depression, anxiety, fatigue, and fibromyalgia on the evaluation of the remission status in patients with rheumatoid arthritis. J Rheumatol. 2014;41(9):1755-60.

8. Ward MM. Are patient self-report measures of arthritis activity confounded by mood? A longitudinal study of patients with rheumatoid arthritis. J Rheumatol. 1994;21(6):1046-50. 
9. Desthieux C, Hermet A, Granger B, Fautrel B, Gossec L. Patient-physician discordance in global assessment in rheumatoid arthritis: a systematic literature review with meta-analysis. Arthritis Care Res (Hoboken). 2016;68:1767-73.

10. Aletaha D, Neogi T, Silman AJ, et al. 2010 Rheumatoid arthritis classification criteria: an American College of Rheumatology/European League Against Rheumatism collaborative initiative. Arthritis Rheum. 2010;62(9):2569-81.

11. Barton JL, Imboden J, Graf J, Glidden D, Yelin EH, Schillinger D. Patient-physician discordance in assessments of global disease severity in rheumatoid arthritis. Arthritis Care Res (Hoboken). 2010;62(6):857-64.

12. Melzack R. The short-form McGill pain questionnaire. Pain. 1987;30(2):191-7.

13. Dworkin RH, Turk DC, Revicki DA, et al. Development and initial validation of an expanded and revised version of the short-form McGill Pain Questionnaire (SF-MPQ-2). Pain. 2009;144(1-2):35-42.

14. Wolfe F, Michaud K, Pincus T. Development and validation of the Health Assessment Questionnaire II: a revised version of the Health Assessment Questionnaire. Arthritis Rheum. 2004;50(10):3296-305.

15. Wolfe F, Clauw DJ, Fitzcharles MA, et al. The American College of Rheumatology preliminary diagnostic criteria for fibromyalgia and measurement of symptom severity. Arthritis Care Res (Hoboken). 2010;62(5):600-10.

16. Wolfe F, Walitt BT, Rasker JJ, Katz RS, Hauser W. The use of polysymptomatic distress categories in the evaluation of fibromyalgia (FM) and FM severity. J Rheumatol. 2015;42(8):1494-501.

17. Nicklin J, Cramp F, Kirwan J, Greenwood R, Urban M, Hewlett S. Measuring fatigue in rheumatoid arthritis: a cross-sectional study to evaluate the Bristol Rheumatoid Arthritis Fatigue multi-dimensional questionnaire, visual analog scales, and numerical rating scales. Arthritis Care Res (Hoboken). 2010;62(11):1559-68.

18. Kroenke K, Spitzer RL, Williams JBW. The PHQ-9: validity of a brief depression severity measure. J Gen Intern Med. 2001;16(9):606-13.

19. Spitzer RL, Kroenke K, Williams JB, Lowe B. A brief measure for assessing generalized anxiety disorder: the GAD-7. Arch Intern Med. 2006;166(10):1092-7.

20. Brown KW, Ryan RM. The benefits of being present: mindfulness and its role in psychological well-being. J Pers Soc Psychol. 2003;84(4):822-48.

21. Ward MM, Guthrie LC, Dasgupta A. Direct and indirect determinants of the patient global assessment in rheumatoid arthritis: differences by level of disease activity. Arthritis Care Res (Hoboken). 2016;(3):323-9. 